\title{
TCGA data mining suggested that high GSDMB expression in bladder cancer indicated an excellent prognosis
}

\author{
Zaichao Xu \\ First Affiliated Hospital of Dalian Medical University \\ Qizhen Tang \\ First Affiliated Hospital of Dalian Medical University \\ Zhiwei Zhang ( $\sim 25406820 @ q q . c o m$ ) \\ First Affiliated Hospital of Dalian Medical University
}

\section{Research Article}

Keywords: GSDMB, bladder cancer, prognosis, TCGA, GSEA

Posted Date: December 18th, 2020

DOI: https://doi.org/10.21203/rs.3.rs-109331/v1

License: (9) This work is licensed under a Creative Commons Attribution 4.0 International License. Read Full License 


\section{Abstract}

Background. Researchers have demonstrated that GSDMB is highly expressed in cancer tissues and located in amplicons, genomic regions that are often amplified during cancer development. We evaluated the role of GSDMB in bladder cancer using publicly available data.

Methods. The relationship between clinical correlation features and GSDMB were analyzed with the Wilcoxon rank sum test (2 groups), Kruskal-Wallis test (multiple groups) and logistic regression. Clinicopathologic characteristics associated with overall survival in TCGA patients using Cox regression and the Kaplan-Meier method. Gene Set Enrichment Analysis (GSEA) was performed using TCGA data set.

Results. GSDMB was differentially expressed between the tumor group and the normal group and was highly expressed in the tumor group. Reduced GSDMB expression in BLCA as significantly associated with grade ( $O R=4.55$, High Grade vs. Low Grade),

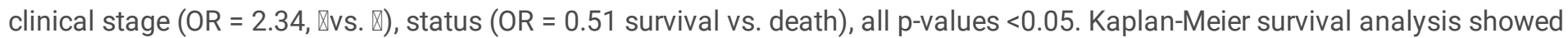
that BLCA with GSDMB- low had a worse prognosis than that with GSDMB-high $(p=0.001)$. The univariate analysis revealed that GSDMB- low correlated significantly with a poor overall survival (OS) (HR: 0.93; 95\%Cl: 0.89-0,98; $p=0.006$ ). The multivariate analysis revealed that GSDMB remained independently associated with overall survival, with a HR of 0.711 (Cl: 0.55-0.92; $p=$ 0.009). GSEA show that focal adhesion, pathways in cancer, small cell lung cancer, renal cell carcinoma, bladder cancer and other cancer related pathways are differentially enriched in GSDMB low expression phenotype.

Conclusions. High GSDMB mRNA expression is an independent risk factor for excellent prognosis in bladder cancer.

\section{Introduction}

GasderminB(GSDMB) is a member of the Gasdermin(GSDM) family and can be regulated by different intramolecular domain interaction mechanisms for lipid binding and pore forming activity [1]. According to previous studies on human cancers, GSDMB is highly expressed not only in healthy tissues (such as lymphocytes, esophagus, stomach, liver, colon, skin epithelium and gastrointestinal tract), but also in gastric cancer, uterine cancer, cervical cancer, breast cancer and other cancer tissues [2,3]. Studies have shown that GSDMB is located in an amplicon, an area of the genome that is often amplified during cancer development. Therefore, GSDMB may play a role in tumor progression and metastasis [1].

Bladder cancer $(\mathrm{BC})$ is a frequently occurring and highly recurrent, fatal malignancy of the genitourinary system, the most common of which is bladder urothelial carcinoma (BUC)[4]. The diagnosis and follow-up of bladder cancer mainly rely on cystoscopy and other means, but this kind of examination will bring some pain to patients, and some patients will interrupt the follow-up due to their intolerance [5]. GSDMB has been found to have significant expression in a variety of tumors. However, the correlation between GSDMB and the prognosis of bladder cancer『Bladder Urothelial Carcinoma『BLCA囚has not been previously reported. Therefore, this study attempted to evaluate the prognostic value of GSDMB expression in human BLCA patients using the data obtained from TCGA (The Cancer Genome Atlas; https://cancergenome.nih.gov/). In order to further understand the biological pathways of the GSDMB regulatory network related to the pathogenesis of BLCA, WE conducted GSEA.

Presently, we demonstrated the effect of GSDMB on prognosis of BLCA, increased GSDMB expression associated with excellent survival in bladder cancer. GSEA show that focal adhesion, pathways in cancer, small cell lung cancer, renal cell carcinoma, bladder cancer and other cancer related pathways are differentially enriched in GSDMB low expression phenotype.

\section{Materials And Methods}

\subsection{RNA-sequencing patient data and bioinformatics analysis.}

The gene expression data (433 cases, normal: tumor=19:414, Workflow Type: HTSeq Counts) and corresponding clinical information were downloaded from TCGA official website. The expression of GSDMB was extracted from the downloaded data, and the data of the normal group and the tumor group were used to draw a scatter plot by applying R (v. 4.0.2) (Fig. 1A). As can be seen from figure, GSDMB expression level in the tumor group was significantly higher than that in the normal group $(P=0.008)$.

Page 2/13 
In tumor and adjacent nontumor tissues of the 434 samples, the expression difference was further analyzed and plotted ( $P$ $=0.022$, Fig. 1B). We reached the same conclusion that GSDMB expression was significantly higher in the tumor group than that in the control group.

Normal BLCA samples were excluded, the samples were divided into the high-expression group and the low-expression group by the median value of GSDMB expression, and the Kaplan-Meier survival curve analysis showed significant difference in the overall survival rate of the groups $(p=0.001$, Fig. $2 A)$.

\subsection{Gene set enrichment analysis}

Gene set enrichment analysis (Gene set enrichment analysis, GSEA) is a kind of calculation method, use the software of the GSEA 4.0.3 to determine whether a priori defined set of genes in a state between two organisms show consistency difference was statistically significant. [6]. A list was first created based on the correlation of all genes with GSDMB expression, and the samples were then divided into the high GSDMB group and the low GSDMB group to identify significant differences in survival. The expression level of GSDMB was used as phenotypic marker, and 1000 genome sequences were performed for each analysis. Nominal phosphorus values and standardized enrichment scores (NES) were used to classify enrichment pathways in each phenotype. Gene sets with a false discovery rate (FDR) $<0.25$ and a normal P-value $<0.05$ were considered significant. GSEA show that focal adhesion, pathways in cancer, small cell lung cancer, renal cell carcinoma, bladder cancer and other cancer related pathways are differentially enriched in GSDMB low expression phenotype.

\subsection{Statistical analysis}

R (v.4.0.2) was used for all statistical analysis. The relationship between patient's clinicopathological features and GSDMB were analyzed with the Wilcoxon rank sum test (2 groups) or Kruskal-Wallis test (multiple groups) and logistic regression.

Clinicopathologic characteristics associated with overall survival in TCGA patients using Cox regression and the Kaplan-Meier method. Data with incomplete clinical information were deleted, univariate Cox analysis selected the factors that may affect prognosis, and multivariate Cox analysis verified the correlation between GSDMB expression and survival and other clinical characteristics. $\mathrm{P}<0.05$ was considered statistically significant. The median value of GSDMB is taken as the threshold value of GSDMB high and low expression.

\section{Results}

\subsection{Patient characteristics}

The clinical data of 433 patients were downloaded from TCGA database (normal: tumor=19:414), including patients' age, gender, histologic grade, clinical stage, TNM classification, and survival status (Table 1).

\subsection{High GSDMB expression in bladder cancer}

GSDMB expression in bladder cancer and normal tissues was compared (Fig. 1A), and the results indicated that GSDMB expression was elevated in bladder cancer $(\mathrm{P}=0.008)$. In the tumor and adjacent nontumor tissues, GSDMB expression in the tumor group was higher than that in the control group $(\mathrm{P}=0.022)$ (Fig. 1B).

\subsection{High Expression of GSDMB in BLCA Is Related to Excellent Overall Survival}

The Kaplan-Meier survival curve analysis showed significant difference in the overall survival rate of the groups ( $p=0.001$, Fig. $2 A)$. BLCA with GSDMB-low had a worse prognosis than that with GSDMB-high. The 5-year survival rate of patients in the highGSDMB expression group (52.4\%) was higher than that of patients in the low-GSDMB expression group (38.1\%).

\subsection{Association with GSDMB expression and clinicopathologic variables.}

A total of 433 BLCA samples with GSDMB expression data across all patient characteristics were analyzed from TCGA. As shown in Fig. 2. ( $B-F)$, Wilcoxon rank sum test(2 groups) or Kruskal-Wallis test(multiple groups) was used to analyze the clinical correlation indicate increased expression of GSDMB correlated significantly with the survival status $(p=1.793 e-6)$, histological 
grade $(p=9.667 e-5)$, clinical stage $(p=0.002), T$ classification $(p=0.002), M$ classification $(p=0.042)$.Univariate analysis using logistic regression revealed that GSDMB expression as a categorical dependent variable was associated with poor prognostic clinicopathologic characteristics (Table 2). Reduced GSDMB expression in BLCA as significantly associated with grade (OR=4.55, High Grade vs. Low Grade), clinical stage (OR=2.34, 『vs. 『), status ( $\mathrm{OR}=0.51$ survival vs. death), all p-values $<0.05$.

\subsection{Univariate and multivariate analyses of correlation between GSDMB expression and OS in bladder cancer patients.}

Univariate analysis showed that low-GSDMB was significantly associated with poor OS (hazard ratio [HR]:0.93; $95 \%$ confidence interval $[\mathrm{Cl}]: 0.89-0.98 ; \mathrm{p}=0.006)$. Other variables associated with poor survival were age, stage, $\mathrm{T}$ classification and $\mathrm{N}$ classification (Table 3). Multivariate analysis showed that GSDMB was independently associated with overall survival, with a HR of 0.71 (Cl: $0.55-0.92, p=0.009$ ) (Table 3, Fig. 3A). Univariate and multivariate Cox analysis indicated that GSDMB expression was an independent risk factor for OS in bladder cancer.

\subsection{The results of GSEA revealed a signal pathway associated with GSDMB}

To identify the activated signaling pathways in bladder cancer, we used GSEA to compare the low-expressed and high-expressed GSDMB datasets. GSEA showed that there were significant differences in the enrichment degree of MSigDBC collection (h.all.v6.2.symbols.gmt), detailed results are shown in Table 4. Gene sets related to focal adhesion, pathways in cancer, small cell lung cancer, renal cell carcinoma, bladder cancer and other cancer related pathways are differentially enriched with the low GSDMB expression phenotype (Fig. 3B-F). Several typical cancer pathways were sketched in Fig. 4A. The KEGG-BLADDERCANCER pathway (NES: -1.9696691, NOM p-val:0, FDR q-val: 0.008737987) were shown in Fig. 4B.

\section{Discussion}

Bladder cancer (BLCA) is the ninth most common cancer worldwide[7]. According to the degree of infiltration, bladder cancer can be divided into non-muscular invasive bladder cancer and muscular invasive bladder cancer[8]. Non-muscular invasive bladder cancer accounts for about $75 \%$ of bladder cancers, $50 \%$ of which are low-level. The main shape of the tumor is micropapillary[9]. The 5 -year survival rate is $90 \%$, but the recurrence rate is high $[10,11]$. Therefore, early diagnosis of BLCA can reduce mortality and improve quality of life. Most current bladder etiology studies focus on genetic changes[12]. Oncogenes are mutated forms of proto-oncogenes that encode growth factors and receptor proteins necessary for normal cell growth[1]. Mutations in protooncogenes can lead to uncontrolled cell division and bladder cancer recurrence and progression. The oncogenes associated with bladder cancer include HER-2, H-RAS, Bcl-2, FGFR3, C-MYC, C-Erbb-2, MDM2, CDC91L1, etc.[10-16] Hypermethylation of SYK, CAGE-1 and other genes has been associated with the progression of bladder cancer[10, 11]. In addition, the occurrence of bladder cancer also includes the amplification or overexpression of normal genes encoding growth factors or their receptors[16, 17], for example, the overexpression of EGEF can increase the aggressiveness and metastasis of bladder cancer[18].

It has been found that GSDMB is highly expressed in various malignant tumors such as gastric cancer, liver cancer and cervical cancer. In different types of cancer in different ways to participate in or affect the occurrence, development, metastasis, drug response and so on $[2,19,20]$. However, the specific function and mechanism of GSDMB in tumorigenesis, progression and metastasis are still unclear[21]. GSDMB expression in bladder cancer囚Bladder Urothelial Carcinoma囚BLCA囚has not been previously reported, We evaluated the role of GSDMB in bladder cancer using publicly available data from The Cancer Genome Atlas (TCGA).

In our study, bioinformatics analysis was performed on data from bladder cancer in TCGA, and the results showed that decreased GSDMB expression in BLCA was associated with clinicopathological characteristics, survival time and poor prognosis. The Kaplan-Meier curve of OS also showed that low GSDMB expression was associated with poor prognosis in bladder cancer patients. Subsequent univariate and multivariate Cox analysis indicated that GSDMB mRNA expression level in bladder cancer patients may be an independent biomarker for prognosis of bladder cancer. In order to further study the function of GSDMB in BLCA, TCGA data was used for GSEA. The results showed that many cancer-related pathways (e.g., bladder, kidney, prostate, pancreas, Table 4) were enriched with the GSDMB low-expression phenotype. GSDMB may be a potential prognostic marker and therapeutic target in BLCA. 
GSDMB expression is associated with cancer prognosis, relapse-free survival, and adverse treatment responses to distant metastases, and GSDMB is also used as a new marker [3, 20,22-26]. In addition, GSDMB may be a potential therapeutic target for cancer therapy [24]. Considering the role of GSDMB in cytotoxic lymphocyte-mediated tumor clearance [20], this analysis may provide guidance for future immunotherapy approaches for cancer. At present, the research of GSDMB in cancer is still unclear, and there is still a large space for the research on the therapeutic targets for the treatment of cancer [27].

\section{Conclusion}

Our analysis showed that GSDMB expression was up-regulated in patients with bladder cancer, and increased GSDMB expression was associated with clinical progression, and was an independent risk factor for OS in patients with bladder cancer. Our findings suggest that GSDMB may be a useful biomarker for prognosis of bladder cancer. The correlation between GSDMB mRNA expression and GSDMB protein expression was not assessed in this report. The specific biological effects of GSDMB need to be verified by further relevant experiments.

\section{Declarations}

\section{Ethics approval and consent to participate}

The data in this paper are from public databases, and do not involve animal or human trials, and do not involve approval by ethics committees.

\section{Consent for publication}

All authors agree to be published

\section{Availability of data and materials}

The data that support the findings of this study are available in TCGA (http: //cancergenome.nih.gov/).

\section{Competing interests}

The authors declare that they have no competing interests.

\section{Funding}

Not applicable

\section{Authors' contributions}

Zaichao Xu and Qizhen Tang wrote the main manuscript text and Zhiwei Zhang prepared figures 1-4. All authors reviewed the manuscript.

\section{Acknowledgments}

The authors extend our deepest gratitude to all the teachers of the Urology Department of the First Affiliated Hospital of Dalian Medical University, who provided assistance in the early stage of manuscript writing. The results shown here are in whole or part based on data generated by the TCGA (http: //cancergenome.nih.gov/).

\section{Abbreviations}

BLCA, bladder urothelial carcinoma; BUC, bladder urothelial carcinoma; Cl, confidence interval; ES, enrichment score; NES, normalized ES; FDR false discovery rate; GSEA, Gene Set Enrichment Analysis; HR, hazard ratio; NOM p-val, normalized p-value; OS, overall survival; TCGA, The Cancer Genome Atlas;

\section{References}

1. Li L, Li Y, Bai Y. Role of GSDMB in Pyroptosis and Cancer. Cancer Manag Res. 2020. 12: 3033-3043. 
2. Crispen PL, Kusmartsev S. Mechanisms of immune evasion in bladder cancer. Cancer Immunol Immunother. 2020. 69(1): 314.

3. Feng S, Fox D, Man SM. Mechanisms of Gasdermin Family Members in Inflammasome Signaling and Cell Death. J Mol Biol. 2018. 430(18 Pt B): 3068-3080.

4. Babjuk M, Burger M, Compérat EM, Gontero P, Mostafid AH, Palou J, van Rhijn B, Rouprêt M, Shariat SF, Sylvester R, Zigeuner R, Capoun O, Cohen D, Escrig J, Hernández V, Peyronnet B, Seisen T, Soukup V. European Association of Urology Guidelines on Non-muscle-invasive Bladder Cancer (TaT1 and Carcinoma In Situ) - 2019 Update. Eur Urol. 2019;76:639-57.

5. Loras A, Martínez-Bisbal MC, Quintás G, Gil S, Martínez-Máñez R, Ruiz-Cerdá JL. Urinary Metabolic Signatures Detect Recurrences in Non-Muscle Invasive Bladder Cancer. Cancers (Basel). 2019. 11(7).

6. Subramanian A, Tamayo P, Mootha VK, et al. Gene set enrichment analysis: a knowledge-based approach for interpreting genome-wide expression profiles. Proc Natl Acad Sci U S A. 2005. 102(43): 15545-50.

7. Mariappan P, Johnston A, Padovani L, et al. Enhanced Quality and Effectiveness of Transurethral Resection of Bladder Tumour in Non-muscle-invasive Bladder Cancer: A Multicentre Real-world Experience from Scotland's Quality Performance Indicators Programme. Eur Urol. 2020.

8. Chen CK, Liao J, Li MS, Khoo BL. Urine biopsy technologies: Cancer and beyond. Theranostics. 2020. 10(17): 7872-7888.

9. Ogawa K, Shimizu Y, Uketa S, Utsunomiya N, Kanamaru S. Prognosis of patients with muscle invasive bladder cancer who are intolerable to receive any anti-cancer treatment. Cancer Treat Res Commun. 2020. 24: 100195.

10. Lin T, Zhang Q, Yuan A, et al. Synergy of Tumor Microenvironment Remodeling and Autophagy Inhibition to Sensitize Radiation for Bladder Cancer Treatment. Theranostics. 2020. 10(17): 7683-7696.

11. Mostafid H, Kamat AM, Daneshmand S, et al. Best Practices to Optimise Quality and Outcomes of Transurethral Resection of Bladder Tumours. Eur Urol Oncol. 2020.

12. Robertson AG, Groeneveld CS, Jordan B, et al. Identification of Differential Tumor Subtypes of T1 Bladder Cancer. Eur Urol. 2020 .

13. Deuker M, Stolzenbach LF, Collà Ruvolo C, et al. Obesity is associated with adverse short-term perioperative outcomes in patients treated with open and robot-assisted radical cystectomy for bladder cancer. Urol Oncol. 2020 .

14. Hua H, Gao Y, Lin J, et al. Quantitative Analysis of Enhanced Computed Tomography in Differentiating Cystitis Glandularis and Bladder Cancer. Biomed Res Int. 2020. 2020: 4930621.

15. Tran T, Melamed J, Deng FM. Gastric adenocarcinoma arising in gastrocystoplasty. Urology. 2020 .

16. van Rhijn B, Mertens LS, Mayr R, et al. FGFR3 Mutation Status and FGFR3 Expression in a Large Bladder Cancer Cohort Treated by Radical Cystectomy: Implications for Anti-FGFR3 Treatment?†. Eur Urol. 2020 .

17. Kohada Y, Hayashi T, Hsi RS, et al. Recurrence and Progression Free Survival of Intermediate Risk Non-Muscle Invasive Bladder Cancer: The impact of Conditional Evaluation and Sub-Classification. BJU Int. 2020 .

18. Choi YW, Yoon HY, Yang HM, et al. Current status of the development of intravesical drug delivery systems for the treatment of bladder cancer. Expert Opin Drug Deliv. 2020 .

19. Ober C, McKennan CG, Magnaye KM, et al. Expression quantitative trait locus fine mapping of the 17q12-21 asthma locus in African American children: a genetic association and gene expression study. Lancet Respir Med. 2020. 8(5): 482-492.

20. Zhou Z, He H, Wang K, et al. Granzyme A from cytotoxic lymphocytes cleaves GSDMB to trigger pyroptosis in target cells. Science. 2020. 368(6494).

21. Chen Q, Shi P, Wang Y, et al. GSDMB promotes non-canonical pyroptosis by enhancing caspase-4 activity. J Mol Cell Biol. 2019. 11(6): 496-508.

22. Ding J, Wang K, Liu W, et al. Pore-forming activity and structural autoinhibition of the gasdermin family. Nature. 2016. 535(7610): 111-6.

23. Molina-Crespo Á, Cadete A, Sarrio D, et al. Intracellular Delivery of an Antibody Targeting Gasdermin-B Reduces HER2 Breast Cancer Aggressiveness. Clin Cancer Res. 2019. 25(15): 4846-4858.

24. Orning P, Lien E, Fitzgerald KA. Gasdermins and their role in immunity and inflammation. J Exp Med. 2019. 216(11): 24532465. 
25. Panoutsopoulou K, Avgeris M, Magkou P, et al. miR-181a overexpression predicts the poor treatment response and earlyprogression of serous ovarian cancer patients. Int J Cancer. 2020.

26. Shi J, Zhao Y, Wang K, et al. Cleavage of GSDMD by inflammatory caspases determines pyroptotic cell death. Nature. 2015. 526(7575): 660-5.

27. Saeki N, Komatsuzaki R, Chiwaki F, Yanagihara K, Sasaki H. A GSDMB enhancer-driven HSV thymidine kinase-expressing vector for controlling occult peritoneal dissemination of gastric cancer cells. BMC Cancer. 2015. 15: 439.

\section{Tables}

Table 1. Clinical characteristics of the bladder cancer patients. 


\begin{tabular}{|c|c|}
\hline Characteristic & $\mathrm{n}(\%)$ \\
\hline \multicolumn{2}{|l|}{ Age } \\
\hline$<60$ years & $88(21.4)$ \\
\hline$\geq 60$ years & $324(78.6)$ \\
\hline \multicolumn{2}{|l|}{ Gender } \\
\hline Female & $108(26.2)$ \\
\hline Male & $304(73.8)$ \\
\hline \multicolumn{2}{|c|}{ Histological grade } \\
\hline High Grade & $388(94.2)$ \\
\hline Low Grade & $21(5.1)$ \\
\hline unknow & $3(0.7)$ \\
\hline \multicolumn{2}{|l|}{ Stage } \\
\hline Stage I & $2(0.5)$ \\
\hline Stage II & 131(31.8) \\
\hline Stage III & $141(34.2)$ \\
\hline Stage IV & 136(33) \\
\hline unknow & $2(0.5)$ \\
\hline \multicolumn{2}{|l|}{ T classification } \\
\hline TO & $1(0.2)$ \\
\hline T1 & $3(0.7)$ \\
\hline T2 & $120(29.1)$ \\
\hline T3 & 196(47.6) \\
\hline $\mathrm{T} 4$ & $59(14.3)$ \\
\hline TX & $1(0.2)$ \\
\hline unknow & $32(7.8)$ \\
\hline \multicolumn{2}{|c|}{$\mathrm{N}$ classification } \\
\hline NO & $239(58)$ \\
\hline N1 & $47(11.4)$ \\
\hline N2 & $76(18.4)$ \\
\hline N3 & $8(1.9)$ \\
\hline NX & $36(8.7)$ \\
\hline unknow & $6(1.5)$ \\
\hline \multicolumn{2}{|c|}{ M classification } \\
\hline Mo & 196(47.6) \\
\hline M1 & $11(2.7)$ \\
\hline$M X$ & $202(49)$ \\
\hline
\end{tabular}

Page $8 / 13$ 


\begin{tabular}{|ll|} 
unknow & $3(0.7)$ \\
\hline Futime & \\
\hline$<1095$ days(3years) & $80(19.4)$ \\
\hline$\geq 1095$ days(3years) & $331(80.3)$ \\
\hline Survival status & \\
\hline Death & $159(38.6)$ \\
\hline Survival & $253(61.4)$ \\
\hline
\end{tabular}

Table 2. GSDMB expression associated with clinical pathological characteristics (logistic regression).

\begin{tabular}{|lllll|}
\hline Clinical characteristics & Total $(\mathrm{N})$ & Odds ratio & $95 \% \mathrm{Cl}(\mathrm{L})$ & $\mathrm{p}$-Value \\
\hline age(continuous) & 409 & 0.985 & $0.99-1.00$ & 0.115 \\
\hline gender (Female vs. Male) & 409 & 1.543 & $0.99-2.43$ & 0.059 \\
\hline grade (High Grade vs. Low Grade) & 406 & 4.550 & $1.65-16.04$ & 0.007 \\
\hline stage (II vs. IV) & 138 & 2.337 & $1.43-3.85$ & 0.001 \\
\hline T (T4 vs. T0) & 377 & 1.000 & $0.00-2015103$ & 1.000 \\
\hline N (N3 vs. N0) & 368 & 0.619 & $0.32-1.77$ & 0.142 \\
\hline M (M1 vs.M0) & 205 & 0.360 & $0.078-1.29$ & 0.140 \\
\hline futime(continuous) & 409 & 1.000 & $0.99-1.00$ & 0.492 \\
\hline
\end{tabular}

$\mathrm{Cl}$, confidence interval. Categorical dependent variable, greater or less than the median expression level.

Table 3. Univariate analysis and multivariate analysis of the correlation of GSDMB expression with OS among bladder cancer patients.

\begin{tabular}{|lllllll|}
\hline Parameter & \multicolumn{2}{l}{ Univariate analysis } & \multicolumn{4}{l|}{ Multivariate analysis } \\
& HR & $95 \% \mathrm{Cl}$ & $\mathrm{p}$-Value & HR & $95 \% \mathrm{Cl}$ & p-Value \\
\hline age & 1.034 & $1.00-1.06$ & 0.020 & 1.022 & $0.99-1.05$ & 0.160 \\
\hline gender & 1.596 & $0.90-2.82$ & 0.107 & 1.615 & $0.90-2.88$ & 0.106 \\
\hline grade & 28316037.779 & $0-$ Inf & 0.996 & 9309078.842 & $0-$ Inf & 0.996 \\
\hline stage & 1.753 & $1.22-2.50$ & 0.002 & 1.248 & $0.57-2.69$ & 0.573 \\
\hline T & 1.646 & $1.11-2.43$ & 0.012 & 1.378 & $0.78-2.42$ & 0.266 \\
\hline N & 1.466 & $1.10-1.94$ & 0.007 & 1.144 & $0.65-1.99$ & 0.637 \\
\hline M & 2.071 & $0.75-5.75$ & 0.163 & 0.934 & $0.28-3.07$ & 0.910 \\
\hline GSDMB & 0.935 & $0.89-0.98$ & 0.006 & 0.711 & $0.55-0.92$ & 0.009 \\
\hline
\end{tabular}

$\mathrm{HR}$, hazard ratio; $\mathrm{Cl}$, confidence interval. 
Table 4. Gene sets enriched in the low GSDMB expression phenotype.

\begin{tabular}{|c|c|c|c|}
\hline NAME & NES & NOM p-val & FDR q-val \\
\hline KEGG_FOCAL_ADHESION & -2.3900921 & 0 & $8.98 \mathrm{E}-04$ \\
\hline KEGG_ECM_RECEPTOR_INTERACTION & -2.3710775 & 0 & 4.49E-04 \\
\hline KEGG_REGULATION_OF_ACTIN_CYTOSKELETON & -2.304208 & 0 & 6.07E-04 \\
\hline KEGG_MELANOMA & -2.288734 & 0 & $4.55 \mathrm{E}-04$ \\
\hline KEGG_GLIOMA & -2.2356787 & 0 & 7.67E-04 \\
\hline KEGG_ARRHYTHMOGENIC_RIGHT_VENTRICULAR_CARDIOMYOPATHY_ARVC & -2.2131152 & 0 & 7.74E-04 \\
\hline KEGG_GAP_JUNCTION & -2.1946673 & 0 & 0.001020793 \\
\hline KEGG_PRION_DISEASES & -2.1657364 & 0 & 0.001146579 \\
\hline KEGG_HYPERTROPHIC_CARDIOMYOPATHY_HCM & -2.138577 & 0 & 0.001326087 \\
\hline KEGG_DILATED_CARDIOMYOPATHY & -2.1257658 & 0 & 0.002220355 \\
\hline KEGG_PATHWAYS_IN_CANCER & -2.1178463 & 0 & 0.002485571 \\
\hline KEGG_GLYCOSAMINOGLYCAN_BIOSYNTHESIS_CHONDROITIN_SULFATE & -2.1052504 & 0 & 0.002751287 \\
\hline KEGG_HEMATOPOIETIC_CELL_LINEAGE & -2.0898116 & 0 & 0.003058765 \\
\hline KEGG_TGF_BETA_SIGNALING_PATHWAY & -2.0648777 & 0 & 0.004456792 \\
\hline KEGG_PATHOGENIC_ESCHERICHIA_COLI_INFECTION & -2.0633962 & 0 & 0.004248562 \\
\hline KEGG_RENAL_CELL_CARCINOMA & -2.059983 & 0 & 0.003983026 \\
\hline KEGG_CELL_ADHESION_MOLECULES_CAMS & -2.0582857 & 0.002057613 & 0.003748731 \\
\hline KEGG_LEUKOCYTE_TRANSENDOTHELIAL_MIGRATION & -2.034828 & 0.002053388 & 0.004881047 \\
\hline KEGG_CHEMOKINE_SIGNALING_PATHWAY & -2.016914 & 0.005940594 & 0.005990018 \\
\hline KEGG_CYTOKINE_CYTOKINE_RECEPTOR_INTERACTION & -2.0038915 & 0.004032258 & 0.006823547 \\
\hline KEGG_BLADDER_CANCER & -1.9980953 & 0 & 0.007112776 \\
\hline KEGG_SMALL_CELL_LUNG_CANCER & -1.9893543 & 0.00408998 & 0.007710421 \\
\hline
\end{tabular}

NES: normalized enrichment score; NOM: nominal; FDR: false discovery rate. Gene sets with NOM p-val $<0.05$ and FDR q-val $<0.25$ are considered as significant.

\section{Figures}



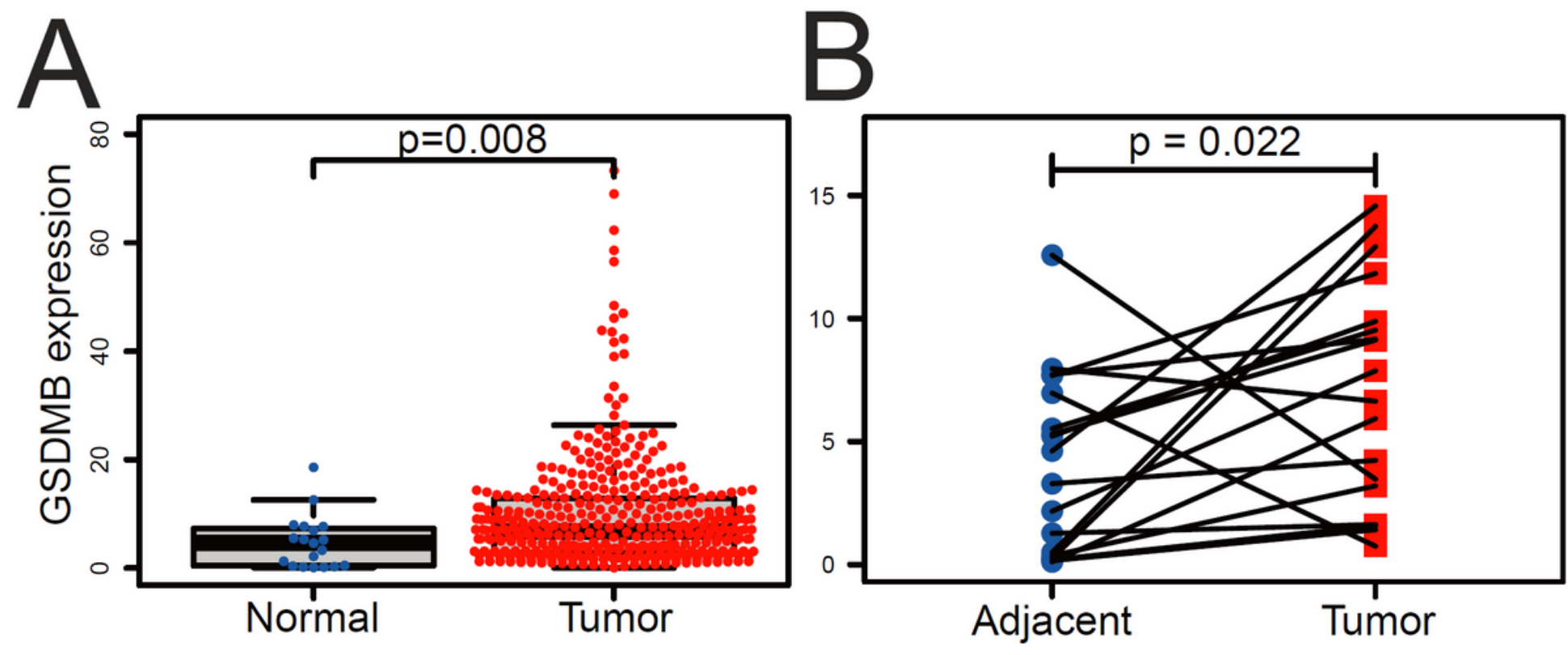

Figure 1

GSDMB expression in bladder cancer. A: GSDMB expression was compared between normal tissues $(\mathrm{N}=19)$ and bladder cancer tissues $(\mathrm{N}=414)$. B: GSDMB expression was compared between tumor and adjacent nontumor tissues.
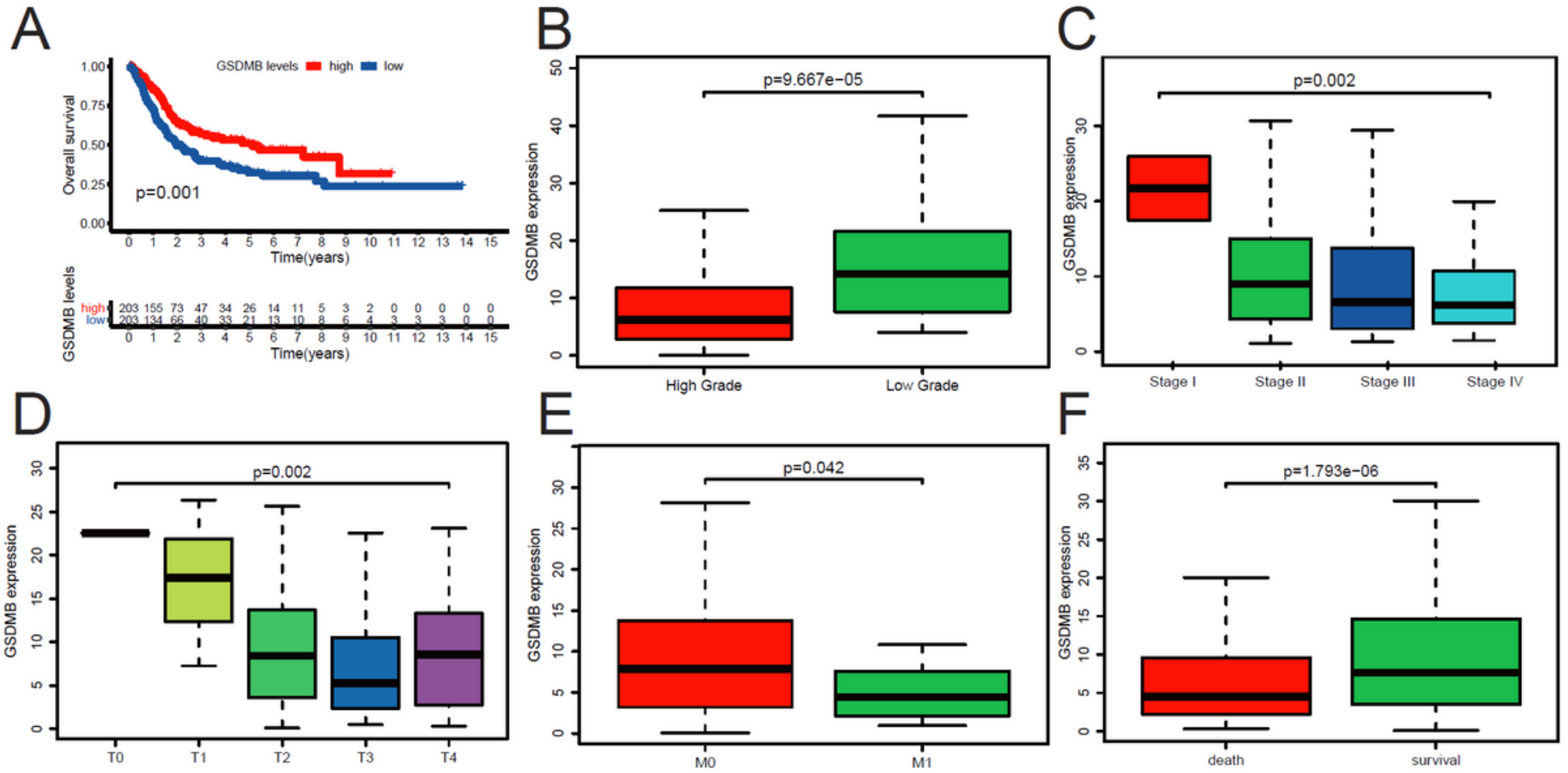

Figure 2

A: Impact of GSDMB expression on overall survival in bladder cancer patients in TCGA cohort. GSDMB expression associated with clinical pathological characteristics in bladder cancer (logistic regression), including B: grade, C: stage, D: T classification, E: M classification, F: survival status. TCGA: The Cancer Genome Atlas. 
A

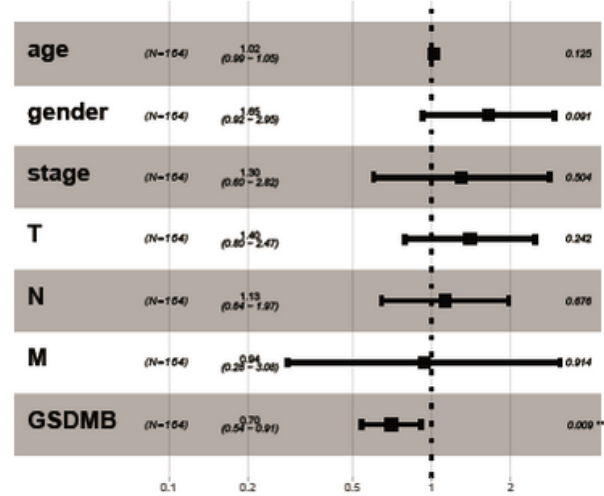

\# Events: 57; Global p-value (Log-Rank): 0.00056092 AIC: 488.64; Concordance Index: 0.7
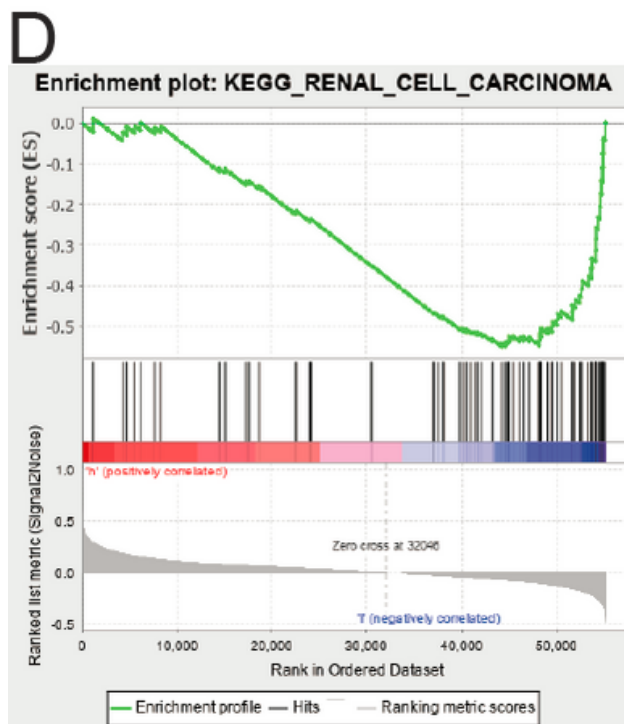

B

Enrichment plot: KEGG_FOCAL_ADHESION

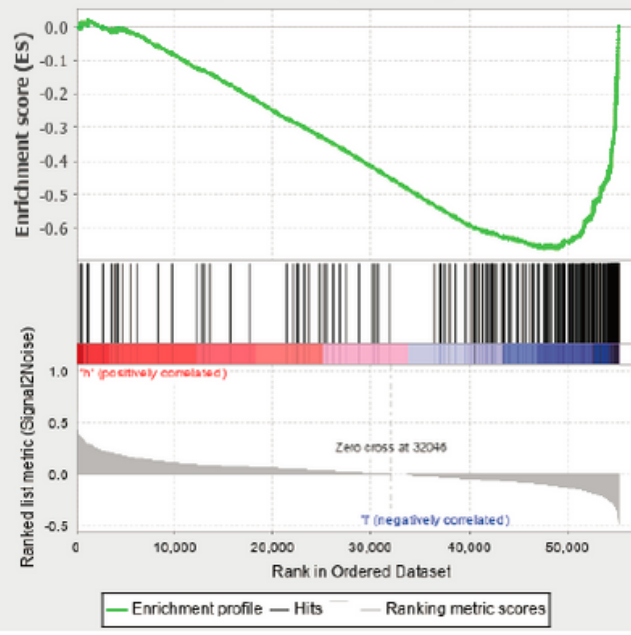

$E$

Enrichment plot: KEGG_SMALL_CELL_LUNG_CANCER
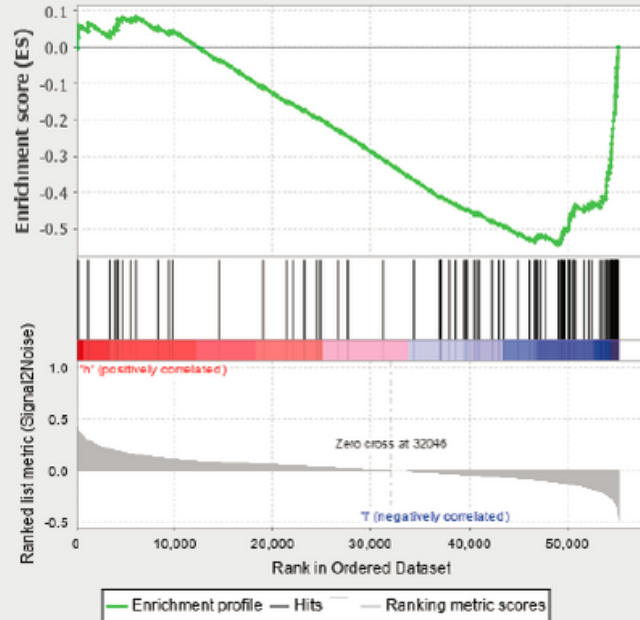

C

Enrichment plot: KEGG_PATHWAYS_IN_CANCER

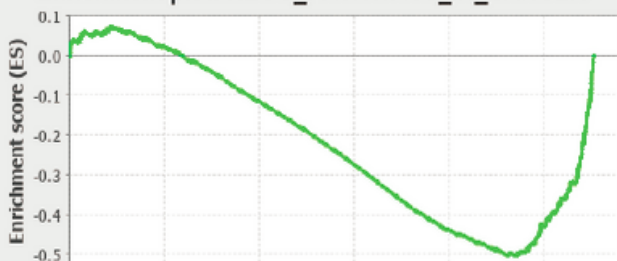

||||||||||||||| ||| ||||||||||||| |||||||||||||||

\$.

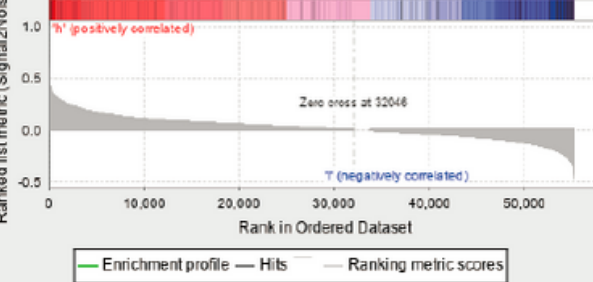

\section{$\mathrm{F}$}

Enrichment plot: KEGG_BLADDER_CANCER

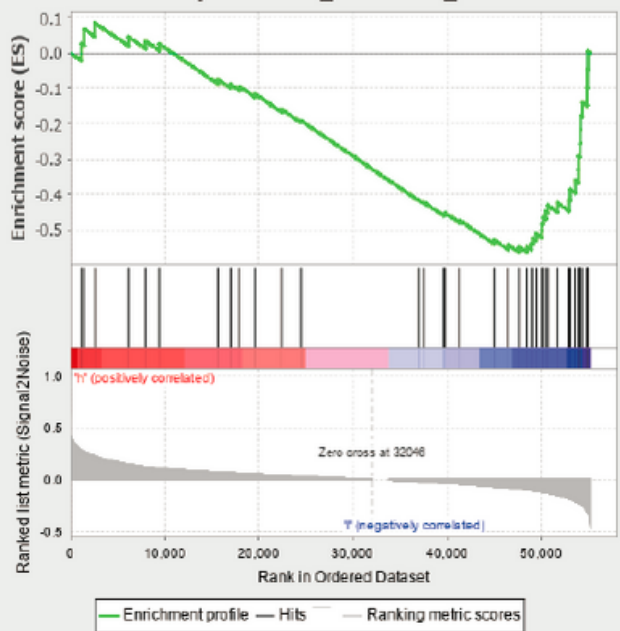

Figure 3

A: Hazard ratio of the correlation of GSDMB expression with OS among bladder cancer patients (multivariate analysis).

Enrichment plots from gene set enrichment analysis (GSEA). GSEA results showing focal adhesion(B), pathways in cancer (C), renal cell carcinoma (D), small cell lung cancer $(E)$, and bladder cancer $(F)$ are differentially enriched in GSDMB-related BLCA. ES, enrichment score; NES, normalized ES; NOM p-val, normalized p-value. 


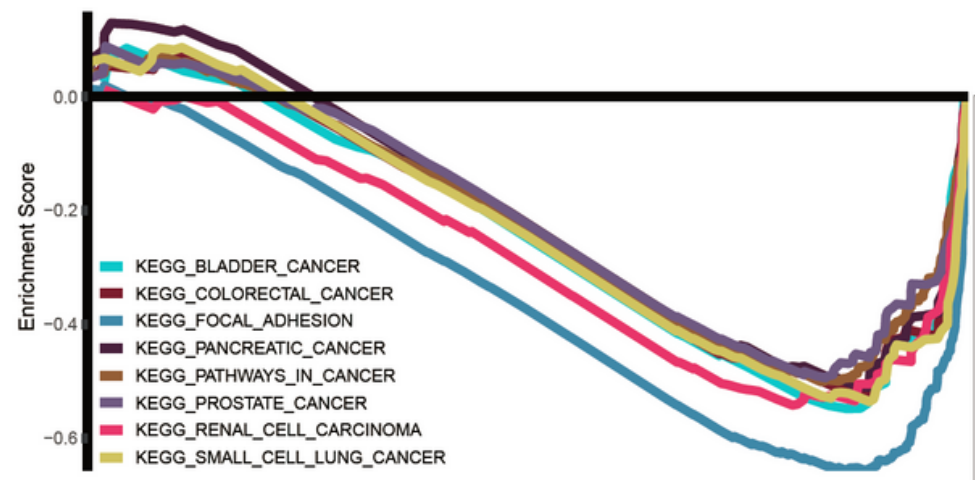

\section{KEGG-BLADDER-CANCER PATHWAY}
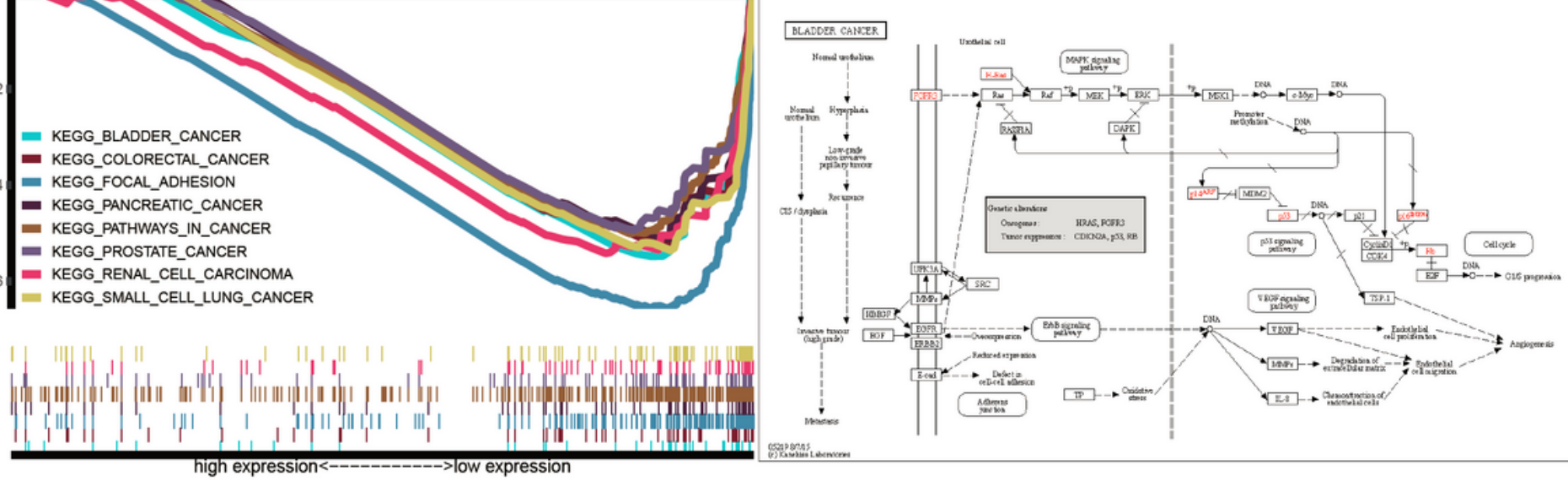

Figure 4

A: GSEA results showing differential enrichment of genes related to cancer pathways in GSDMB-related BLCA.B: The KEGGBLADDER-CANCER pathway (NES: -1.9696691, NOM p-val:0, FDR q-val: 0.008737987). KEGG: Kyoto Encyclopedia of Genes and Genomes. 